\title{
EL DICTAMEN DEL CONSEJO DE ESTADO 24 DE 13 DE MARZO DE 2008, SOBRE EL TRATADO DE LISBOA
}

\author{
SUMARIO \\ I. Competencia del Consejo de Estado para \\ emitir el dictamen \\ II. Los antecedentes \\ III. Las consideraciones del dictamen
}

\section{COMPETENCIA DEL CONSEJO DE ESTADO PARA EMITIR EL DICTAMEN}

La Comisión Permanente del Consejo de Estado ha aprobado en Sesión de 13 de marzo de 2008 el dictamen $n^{\circ}$ 24/2008, relativo al Tratado de Lisboa, por el que se modifican el Tratado de la Unión Europea y los Tratados constitutivos de la Comunidad Europea, hecho en Lisboa el 13 de diciembre de 2007.

El artículo 22.1 de la Ley Orgánica del Consejo de Estado, de 22 de abril de 1980, prescribe la consulta a su Comisión Permanente «en todos los tratados o convenios internacionales sobre la necesidad dc autorización de las Cortes Generales con carácter previo a la prestación del consentimiento del Estado". Los dictámenes del Consejo de Estado emitidos en cumplimiento de dicha previsión se circunscriben a la determinación del procedimiento que ha de seguirse para formar la voluntad del Estado, sin que se entre en el examen técnico- jurídico de los textos que son objeto de la consulta. Ello exige la calificación del tratado a los efectos del artículo 94.1 de la Constitución que prevé que la prestación del consentimiento del Estado para obligarse por medio de tratados o convenios requerirá la previa autorización de las Cortes Generales en los casos de: tratados de carácter político (a), tratados o convenios de carácter militar (b), tratados o convenios que afecten a la integridad territorial del Estado o a los derechos y deberes fundamentales establecidos en el Título 1 (c), tratados o convenios que impliquen obligaciones financieras para la Hacienda Pública (d) y tratados o convenios que supongan la modificación o derogación de una Ley o exijan medidas legislativas para su ejecución (e). Si el tratado no es 
subsumible en ninguno de tales supuestos el artículo 94.2 de la Constitución prescribe que el Congreso de los Diputados y el Senado serán inmediatamente informados en la conclusión de los restantes tratados o convenios.

Cabe la posibilidad de que la autorización de las Cortes Generales requiera una ley orgánica. Es el supuesto del artículo 93 de la Constitución, según el cual, mediante ley orgánica se podrá autorizar la celebración de tratados por los que se atribuya a una organización o institución internacional el ejercicio de competencias derivadas de la Constitución. También ha de tenerse presente el artículo 95 de la Constitución, de acuerdo con el cual "la celebración de un tratado internacional que contenga estipulaciones contrarias a la Constitución exigirá la previa revisión constitucional. El Gobierno o cualquiera de las Cámaras puede requerir al Tribunal Constitucional para que declare si existe o no esa contradicción".

Este es el marco normativo dentro del cual el Consejo de Estado interviene en relación con todos los tratados o convenios internacionales que el Gobierno se propone concluir (o en relación con formulación de reservas, denuncias y declaraciones interpretativas) a fin de determinar si es necesaria o no la autorización de las Cortes Generales con carácter previo a la prestación del consentimiento en obligarse.

\section{LOS ANTECEDENTES}

El dictamen del Consejo de Estado sobre el Tratado de Lisboa dedica la primera parte de los antecedentes a exponer la estructura y los principales rasgos de dicho Tratado. El resto de los antecedentes se dedica a exponer el contenido de los informes que figuran en el expediente y del infonne con propuesta de resolución elaborado por la División de Tratados Internacionales del Ministerio de Asuntos Exteriores y de Cooperación que considera que la prestación del consentimiento del Estado para ratificar el Tratado de Lisboa deber ser autorizada mediante Ley Orgánica, de acuerdo con lo previsto en el artículo 93 de la Constitución, al igual que sucedió en el caso de la adhesión de España a las Comunidades Europeas y en los de ratificación del Acta Única Europea y de los Tratados de Maastricht, Amsterdam y Niza (lo mismo se entendió en el Tratado por el que se establece una Constitución para Europa). Resulta clarificador recoger la estructura y algunos de los aspectos del contenido del Tratado que figuran en el dictamen de referencia.

\section{II.1 Estructura DEL Tratado De LisboA}

El Tratado de Lisboa por el que se modifican el Tratado de la Unión Europea y los Tratados constitutivos de la Comunidad Europea, hecho en Lisboa el 13 de diciembre de 2007 y publicado en el Diario Oficial de la Unión Europea el 17 de diciembre de 2007, consta de un preámbulo, siete artículos, 
once Protocolos que deberán ir anejos al Tratado de la Unión Europea, al Tratado de Funcionamiento de la Unión Europea y, en su caso, al Tratado constitutivo de la Comunidad Europea de la Energía Atómica, dos Protocolos que deberán ir anejos al Tratado de Lisboa y un Anexo. El Acta Final de la Conferencia Intergubernamental incluye 43 Declaraciones relativas a disposiciones de los Tratados, 7 Declaraciones relativas a los Protocolos de los Tratados y 15 Declaraciones de Estados miembros.

El Tratado de Lisboa no deroga los tratados comunitarios actualmente vigentes para establecer un nuevo texto único, como hacía el Tratado por el que se establece una Constitución para Europa y que no llegó a entrar en vigor por falta de conclusión de su proceso de ratificación en los Estados miembros, sino que los enmienda. Se sigue, pues, el procedimiento clásico de revisión de un tratado. Así el Tratado de la Unión Europea (TUE) conservará su denominación actual y contendrá los aspectos fundamentales de la Unión Europea, será una suerte de tratado básico. El Tratado constitutivo de la Comunidad Europea pasará a llamarse Tratado de Funcionamiento de la Unión Europea (TFUE) y regulará el funcionamiento de la Unión en lo que atañe a las instituciones, procedimientos y competencias, entre otros aspectos. Como determina en su artículo 1 en la renumeración efectuada por el anexo, "organiza el funcionamiento de la Unión y determina los ámbitos, la delimitación y las condiciones de ejercicio de sus competencias". Ambos Tratados tendrán, en todo caso, el mismo valor jurídico.

El resultado de esta operación modificativa no está exento de complejidad dada la introducción de muchos artículos con un mismo número (diferenciados por la secuencia de ordinales en latín) para ampliar y desarrollar la regulación de algunas materias, la supresión de numerosos artículos que quedan sin contenido o el desplazamiento de múltiples preceptos. El Anexo, de conformidad con el artículo 5 del Tratado de Lisboa renumera mediante unas tablas de correspondencias los artículos, secciones, capítulos y partes del TUE y del TCE, por lo que constituye una solución práctica idónea para una racionalización estructural y mejor comprensión sistemática de los Tratados, que cristaliza con la versión consolidada oficial de éstos (de momento, hasta la entrada en vigor del Tratado de Lisboa, existe una versión provisional consolidada de los dos Tratados en el Diario Oficial de la Unión Europea de 9 de mayo de 2008).

El preámbulo, muy breve, indica en dos párrafos que los Estados miembros desean completar el proceso iniciado por el Tratado de Amsterdam y el Tratado de Niza con el fin de reforzar la eficacia y la legitimidad democrática de la Unión y mejorar la coherencia de su acción, por lo que convienen en modificar el Tratado de la Unión Europea, el Tratado constitutivo de la Comunidad Europea y el Tratado constitutivo de la Comunidad Europea de la Energía Atómica.

El articulado del Tratado de Lisboa se inicia con dos extensos artículos, el primero referido a las modificaciones al Tratado de la Unión Europea (TUE) y el segundo a las modificaciones al Tratado constitutivo de la Comunidad Eu- 
ropea (TCE). El TUE tendrá 55 artículos con la renumeración que efectúa el Anexo, que se estructurarán en 6 títulos: disposiciones comunes, disposiciones sobre los principios democráticos, disposiciones sobre las instituciones, disposiciones sobre las cooperaciones reforzadas, disposiciones generales relativas a la acción exterior de la Unión y disposiciones específicas relativas a la política exterior y disposiciones finales. El TFUE tendrá 358 artículos según la nueva numeración que da el Anexo, encuadrados en siete partes: principios, no discriminación y ciudadanía de la Unión, políticas y asociaciones internas de la Unión, asociación de los países y territorios de ultramar, acción exterior de la Unión, disposiciones institucionales y financieras y disposiciones generales y finales.

Las disposiciones finales (artículos 3 a 7) se refieren a las siguientes cuestiones:

— La duración del Tratado será por un periodo de tiempo ilimitado (artículo 3).

- El Protocolo $\mathrm{n}^{\circ} 1$ anejo al Tratado contiene las modificaciones de los Protocolos anejos al TUE, al Tratado constitutivo de la Comunidad Europea y/o al Tratado constitutivo de la Comunidad Europea de la Energía Atómica. El Protocolo ${ }^{\circ} 2$ anejo al Tratado contiene las modificaciones al Tratado constitutivo de la Comunidad Europea de la Energía Atómica (artículo 4).

- Los artículos, secciones, capítulos, títulos y partes del TUE y del TCE modificados por el Tratado se numeran de nuevo de conformidad con las tablas de correspondencias que figuran en el Anexo al Tratado, que es parte integrante de éste, debiendo seguirse en las referencias que se hagan la nueva numeración (artículo 5).

- El Tratado será ratificado por las Altas Partes Contratantes, de conformidad con sus respectivas normas constitucionales. Los instrumentos de ratificación serán depositados ante el Gobierno de la República Italiana. El Tratado entrará en vigor el 1 de enero de 2009, siempre que se hayan depositado todos los instrumentos de ratificación o, en su defecto, el primer día del mes siguiente al del depósito del instrumento de ratificación del último Estado signatario que cumpla dicha formalidad (artículo 6).

- El artículo 7 dispone que los textos del tratado en las lenguas oficiales de los Estados miembros de la Unión son igualmente auténticos y el ejemplar único en que se hace será depositado en los archivos del Gobierno de la República Italiana, que remitirá una copia autenticada a cada uno de los Gobiernos de los demás Estados signatarios.

Los Protocolos que deberán ir anejos al TUE, al Tratado de Funcionamiento de la UE y, en su caso, al Tratado constitutivo de la Comunidad Europea de la Energía Atómica versan sobre los siguientes aspectos: cometido de los Parlamentos nacionales en la Unión Europea; la aplicación de los princi- 
pios de subsidiariedad y proporcionalidad; Eurogrupo; cooperación estructurada permanente establecida por el artículo 28.A del Tratado de la Unión Europea; apartado 2 del artículo 6 del Tratado de la Unión Europea relativo a la adhesión de la Unión al Convenio para la Protección de los Derechos $\mathrm{Hu}-$ manos y de las Libertades Fundamentales; mercado interior y competencia; ejercicio de las competencias compartidas; servicios de interés general; decisión del Consejo relativa a la aplicación del apartado 4 del artículo 9 c del TUE y del apartado 2 del artículo 205 del TFUE entre el 1 de noviembre de 2014 y el 31 de marzo de 2017, por una parte, y a partir del 1 de abril de 2017, por otra y disposiciones transitorias.

Por otra parte, hay dos Protocolos que deberán ir anejos al Tratado de Lisboa. El $n^{\circ} 1$, por el que se modifican los Protocolos anejos al TUE, TCE y/o al Tratado constitutivo de la Comunidad Europea de la Energía Atómica, seguido de un anexo con las tablas de correspondencias a que se refiere el artículo 2 del mismo Protocolo $n^{\circ} 1$. El Protocolo $n^{\circ} 2$, por el que se modifica el Tratado constitutivo de la Comunidad Europea de la Energía Atómica.

El Anexo al Tratado de Lisboa, que sigue a los Protocolos, incluye las tablas de correspondencias a que se refiere el artículo 5 del Tratado de Lisboa.

\section{II.2 Contenido Del TRATADo DE LisboA}

El dictamen 24/2008 al exponer el contenido de las modificaciones que se incorporan a los tratados TUE y TCE distingue entre diversas materias.

A. En primer lugar se adiciona un párrafo tercero al Tratado de la Unión Europea que establece que «La Unión se fundamenta en el presente Tratado y en el Tratado de Funcionamiento de la Unión Europea (en lo sucesivo denominados "los Tratados"). Ambos Tratados tienen el mismo valor jurídico. La Unión sustituirá y sucederá a la Comunidad Europea".

Por otra parte, desaparece la actual estructura de la Unión en tres pilares (un pilar comunitario - CE y CEEA- y los dos pilares extracomunitarios o intergubernamentales de la Política Exterior y de Seguridad Común- PESC- y de la Cooperación Policial y Judicial en materia Penal) para determinarse que la estructura de la Unión Europea es única ( sin perjuicio de que los mecanismos de decisión, según el caso, varíen desde los propios de la integración a los de simple cooperación).

Como modificaciones horizontales en el que se denominará Tratado de Funcionamiento de la Unión Europea, se dispone que las palabras "la Comunidad" o "la Comunidad Europea" se sustituyan por "la Unión", las palabras "de las Comunidades Europeas" O "de la CEE" se sustituyan por "de la Unión Europea", y los adjetivos "comunitario", "Comunitaria", "Comunitarios" y "comunitarias" se sustituyan por "de la Unión" con carácter general.

B. En cuanto a valores, principios y derechos de la Unión, el Tratado de Lisboa — que pierde el preámbulo del Tratado Constitucional— añade sin embargo, al preámbulo del TUE un nuevo párrafo, referido a la inspiración "en la 
herencia cultural, religiosa y humanista de Europa, a partir de la cual se han desarrollado los valores universales de los derechos inviolables e inalienables de la persona, así como la libertad, la democracia, la igualdad y el Estado de Derecho".

El nuevo artículo 1 bis del TUE (que será renumerado como artículo 2) establece que «la Unión se fundamenta en los valores de respeto de la dignidad humana, libertad, democracia, igualdad, Estado de Derecho y respeto de los derechos humanos, incluidos los derechos de las personas pertenecientes a minorías. Estos valores son comunes a los Estados miembros en una sociedad caracterizada por el pluralismo, la no discriminación, la tolerancia, la justicia, la solidaridad y la igualdad entre mujeres y hombres".

El Tratado de Lisboa conserva y consolida las cuatro libertades del mercado e incluye la solidaridad como un valor esencial entre los Estados miembros (en caso de ataque terrorista, catástrofe natural o de origen humano así como en el sector de la energía, según la cláusula de solidaridad que constituye el nuevo artículo 222 del TFUE y su artículo 194, referido a la energía). La libre competencia deja de estar entre los objetivos de la Unión para el funcionamiento del mercado interior, si bien su regulación aparece deferida a un Protocolo ad hoc al TUE y al TFUE.

Los nuevos artículos 3 bis y 3 ter del TUE establecen y desarrollan los principios de atribución de competencias, subsidiariedad, proporcionalidad y cooperación leal entre la Unión y los Estados miembros.

No figura en la parte dispositiva del Tratado referencia expresa al principio de primacía del Derecho de la Unión Europea, siendo sustituida ahora la previsión que hacía el Tratado por el que se establece una Constitución para Europa por una Declaración que se limita a recordar "que, con arreglo a la jurisprudencia reiterada del Tribunal de Justicia de la Unión Europea, los Tratados y el Derecho adoptado por la Unión sobre la base de los mismos priman sobre el Derecho de los Estados miembros, en las condiciones establecidas por la citada jurisprudencia". Asimismo, la Conferencia Intergubernamental incorporó al Acta Final el dictamen del Servicio Jurídico del Consejo sobre la primacía, en el que se dice que "el principio de la primacía del Derecho Comunitario es inherente a la naturaleza específica de la Unión Europea".

Por lo que se refiere a la Carta de Derechos Fundamentales de la Unión Europea, a diferencia del Tratado Constitucional, no resulta incorporada al texto del Tratado de Lisboa. El artículo 6.1 del TUE tendrá un primer apartado, según el cual, «la Unión reconoce los derechos, libertades y principios enunciados en la Carta de los Derechos Fundamentales de 7 de diciembre de 2000, tal como fue adoptada el 12 de diciembre de 2007 en Estrasburgo, la cual tendrá el mismo valor jurídico que los Tratados". Los problemas que suscitó la pretendida inclusión de la Carta en el texto del Tratado tienen reflejo en el Protocolo relativo a la aplicación de la Carta de los Derechos Fundamentales al Reino Unido y a Polonia y en dos Declaraciones de Polonia en relación con la Carta citada. A todo ello hay que añadir, en la misma línea que el Tratado 
Constitucional, que el futuro apartado 2 del artículo 6 del TUE establece que «la Unión se adherirá al Convenio Europeo para la Protección de los Derechos Humanos y de las Libertades Fundamentales. Esta adhesión no modificará las competencias de la Unión que se definen en los Tratados". El apartado 3 añade que los derechos fundamentales que garantiza el Convenio y los que son fruto de las tradiciones constitucionales comunes a los Estados miembros formarán parte del Derecho de la Unión como principios generales. El Protocolo sobre el apartado 2 del artículo 6 del TUE precisa los términos de la adhesión.

C. En el plano institucional el Tratado de Lisboa no altera de forma esencial la arquitectura institucional ya existente de la Unión, si bien introduce algunas novedades con el fin de incrementar su eficacia y transparencia: se reconoce a la Unión Europea personalidad jurídica (artículo 47 TUE renumerado) que desplegará amplios efectos en el ámbito de las relaciones internacionales; el Parlamento Europeo es reforzado en sus competencias, en materia legislativa, presupuestaria y de tratados internacionales, y el procedimiento de codecisión se convierte en el procedimiento legislativo ordinario, situándose casi en situación de paridad respecto del Consejo; la institucionalización del Consejo Europeo y la previsión de que éste tendrá un Presidente; la generalización de la mayoría cualificada en el Consejo como regla de decisión para gran número de políticas (a partir del 1 de noviembre de 2014 la mayoría cualificada seguirá el principio de doble mayoría — de los Estados miembros y de la población- que refleja la legitimidad dual de la Unión); las modificaciones institucionales en la composición de la Comisión y del Parlamento para agilizar su funcionamiento en una Unión ampliada (a partir del 1 de noviembre de 2014 reducirá el número de sus integrantes y su composición se regirá por un principio de rotación igual entre los Estados miembros); la creación del puesto de Alto Representante de la Unión Europea para Asuntos Exteriores y Política de Seguridad, que será asistido por un nuevo Servicio Europeo de Acción Exterior; en relación con el Tribunal de Justicia de la Unión Europea, que comprenderá el Tribunal de Justicia, el Tribunal General (antes de Primera Instancia) y los tribunales especializados, se amplía el ámbito de su jurisdicción en particular a la cooperación en materia penal y a la cooperación policial, aunque seguirá quedando fuera la PESC, y se introducen algunas modificaciones de procedimiento; el Banco Central Europeo y el Tribunal de Cuentas pasan a ser considerados como instituciones de la Unión, elevándose su número a siete.

D. Por lo que se refiere a los actos de la Unión, se mantienen las categorías clásicas de reglamentos y directivas (frente a las "leyes" y "leyes marco" del Tratado Constitucional), así como decisiones, recomendaciones y dictámenes. Se introduce una clasificación en actos legislativos, actos delegados y actos de ejecución, en línea de continuidad con las previsiones de aquel Tratado.

E. Para la adopción de los actos legislativos, el procedimiento de codecisión pasa a llamarse procedimiento legislativo ordinario, ampliándose a varios ámbitos como la inmigración legal, la cooperación judicial penal (Euro- 
just, prevención de la delincuencia, aproximación de las normas penales, infracciones y sanciones), la cooperación policial (Europol) o algunas disposiciones de la política comercial o agrícola. El Parlamento Europeo deberá aprobar el marco financiero plurianual, con el Consejo deberá fijar todos los gastos de manera conjunta y deberá aprobar todos los acuerdos internacionales en aquellos asuntos que correspondan al procedimiento legislativo ordinario. Los Parlamentos nacionales cobran un papel más relevante con el Tratado de Lisboa para participar en las tareas de la Unión. Se establecen sus derechos y obligaciones en cuanto a su información, el control del principio de subsidiariedad, los mecanismos de evaluación en el espacio de libertad, seguridad y justicia así como en lo que se refiere a la revisión de los Tratados. Se introduce la "iniciativa ciudadana", reconociéndose que un grupo al menos de un millón de ciudadanos de un número significativo de Estados miembros podrá pedir a la Comisión que formule propuestas de legislación en los ámbitos de competencia de la Unión.

F. En el plano competencial, se clarifica el reparto de competencias entre la Unión Europea y sus Estados miembros (siguiendo la clasificación que hacía el Tratado Constitucional) distinguiéndose entre competencias exclusivas, compartidas y acciones de apoyo, coordinación o complemento. Se amplia el ámbito de aplicación de las cooperaciones reforzadas que se regulan en los artículos 20 del TUE y 326 a 334 del TFUE renumerados.

G. En materia de políticas, el Tratado de Lisboa regula la acción exterior de la Unión y deja de ser un pilar comunitario. La PESC se regirá por reglas y procedimientos específicos; será definida por el Consejo Europeo y el Consejo, que deberán pronunciarse por unanimidad salvo cuando los Tratados dispongan otra cosa. Queda excluida en este ámbito la adopción de actos legislativos. Se incluye en el ámbito de la Unión lo que se ha llamado "Tercer Pilar" relativo a la cooperación policial y judicial en materia penal. El Tratado de Lisboa introduce nuevas políticas, como la espacial y la de propiedad intelectual. Se revalida el compromiso de la UE para la elaboración de una política de inmigración común. Se refuerzan algunas de las políticas existentes como es el caso en la política de energía de la introducción de la noción de solidaridad o en el de la política de medio ambiente de la inclusión por primera vez de la lucha contra el cambio climático entre sus objetivos. En virtud de la "cláusula social", los aspectos sociales deben ser tenidos en cuenta al definir y ejecutar todas las políticas. Se establece una base jurídica específica para la ayuda humanitaria y se contempla la posibilidad de creación de un Cuerpo Voluntario de Ayuda Humanitaria.

\section{LAS CONSIDERACIONES DEL DICTAMEN}

Las consideraciones del dictamen se dividen en cuatro apartados que finalizan con la conclusión en la que se afirma que "la prestación del consentimiento del Estado para obligarse mediante el Tratado de Lisboa por el que 
se modifican el Tratado de la Unión Europea y los Tratados constitutivos de la Comunidad Europea, firmado el 13 de diciembre de 2007, requiere autorización de las Cortes Generales mediante ley orgánica aprobada al amparo del artículo 93 de la Constitución".

\section{III.1 LA SIGNIFICACIÓN DEL TRATADO DE LISBOA}

Las consideraciones del dictamen comienzan haciendo referencia a la competencia del Consejo de Estado en cuya virtud se emite la consulta (artículo 22.1 de la Ley Orgánica del Consejo de Estado) para continuar expresando las circunstancias en que se ha originado el Tratado de Lisboa y su significación.

Destaca el Consejo de Estado que, frente a la vía que se siguió en la génesis del non nato Tratado por el que se establece una Constitución para Europa, el Tratado de Lisboa representa la búsqueda de una salida a la crisis en que se sumió la Unión Europea tras el fallido proceso de ratificación del primero mediante la vuelta a la técnica clásica de revisión de los tratados integrantes del Derecho primario y a través del aligeramiento de ciertos contenidos de aquél. Si con la fórmula "constitucional", producto de una Convención ad hoc, se pretendió en cierto modo una refundación de la Unión (según el artículo IV-43 8.1 del Tratado Constitucional «la Unión Europea creada por el presente Tratado sucede a la Unión Europea constituida por el Tratado de la Unión Europea y a la Comunidad Europea"), con el Tratado de Lisboa - menos ambicioso en sus pretensiones formales al tratarse de un tratado de reforma- se retorna al método que establecieron y siguieron los fundadores de las Comunidades Europeas en sus primeros pasos. Así, el Tratado de Lisboa, tal como indica su propio preámbulo — que no menciona al Tratado por el que se establece una Constitución para Europa-, se sitúa en la estela de los Tratados de Amsterdam y de Niza, viniendo a completar el proceso iniciado por éstos con el fin de reforzar la eficacia y legitimidad democrática de la Unión y mejorar la coherencia de su acción.

El relanzamiento del proceso de integración tras el fracaso de la Constitución para Europa se produjo dos años después de los referenda negativos por parte de Francia y de los Países Bajos a la ratificación del Tratado por el que se establecía aquélla. La Presidencia alemana puso la primera piedra con la Declaración de Berlín de 25 de marzo de 2007 — para conmemorar el 50 aniversario de los Tratados de Roma- y el Consejo Europeo de 21 y 22 de junio de 2007 alcanzó un acuerdo que suponía un mandato de convocatoria de una nueva Conferencia Intergubernamental, mandato que constituía "la base y el marco exclusivo de la labor de la Conferencia Intergubernamental" y que era sumamente preciso y detallado. El Parlamento Europeo, la Comisión y el Banco Central Europeo emitieron los preceptivos informes requeridos por el Consejo en julio de 2007 y mediante Resolución de 11 de julio de 2007 se convocó a la CIG. La negociación del nuevo Tratado habría de hacerse en un 
año y medio, con un estrecho margen negociador, a través del clásico procedimiento de revisión mediante una CIG de carácter diplomático (que celebró cerca de treinta reuniones de expertos jurídicos y tres de los Ministros).

En la senda de las reformas de los Tratados constitutivos operadas por el Acta Única Europea (1986), el Tratado de Maastricht (1992), el Tratado de Amsterdam (1997) y el Tratado de Niza (2201), el Tratado de

Lisboa nace como el último eslabón por el momento del proceso abierto de integración que ha supuesto la progresiva ampliación de las competencias atribuidas por los Estados miembros originariamente a las Comunidades Europeas y después a la Unión. Y, aunque no produce el salto cualitativo que implicaba el Tratado Constitucional en el plano político, la culminación satisfactoria del proceso de ratificación en curso habrá de representar un avance de gran relevancia en la historia europea.

En realidad, se ha optado por la vía de un Tratado de reforma que acoge en su seno una gran parte del Tratado Constitucional, dejando en el camino aquellos elementos que generaban rechazo en algunos Estados por su excesiva connotación constitucional o estatal, sobre todo en su resonancia más formal que material. El mandato que recibió la CIG era taxativo en cuanto a que el resultado de la revisión "no tendrá carácter constitucional", en los Tratados resultantes "no se utilizará el término Constitución" y "no figurará en los Tratados modificados ningún artículo que mencione los símbolos de la Unión (bandera, himno o divisa). Así, han decaído las referencias a los símbolos de la Unión, al Ministro de Asuntos Exteriores, a las leyes y leyes marco, al principio de primacía del Derecho de la Unión Europea sobre los ordenamientos internos) y se han introducido nuevas cautelas a favor de las competencias estatales. No obstante lo anterior, un numeroso grupo de Estados (Bélgica, Bulgaria, Alemania, Grecia,. España, Italia, Chipre, Lituania, Luxemburgo, Hungría, Malta , Austria, Portugal, Rumanía, Eslovenia y Eslovaquia) adoptaron una declaración a través de la que afirmaron que la bandera, el himno, el euro y la divisa "seguirán siendo, para ello, los símbolos de la pertenencia común de los ciudadanos a la Unión Europea y de su relación con ella".

Considera el Consejo de Estado que, si el Tratado de Lisboa ha seguido fielmente en lo formal el procedimiento de revisión previsto en el Derecho primario, en cambio en el orden material retiene una gran parte de las innovadoras reformas que preveía el Tratado Constitucional, a los efectos de dotar a la Unión de más eficacia en la toma de decisiones, más democracia gracias al mayor protagonismo del Parlamento Europeo y los Parlamentos nacionales y más coherencia en la acción exterior. Ha rescatado, así, selectivamente las más significativas aportaciones que el Tratado Constitucional efectuaba en cuanto a instituciones, procedimientos y competencias, con correcciones de estilo varias y redistribuyendo las novedades — sobre todo las de la Parte IIIdel Tratado Constitucional a lo largo y ancho del texto del TUE y, fundamentalmente, del TFUE.

Añade el Consejo de Estado que el desprendimiento de la pátina constitucional que se opera en virtud del Tratado de Lisboa por comparación con el 
previo Tratado relativo al establecimiento de una Constitución para Europa y la menor transparencia en su elaboración ha sido la fórmula útil —aunque no exenta de críticas - arbitrada para cohonestar las muy distintas sensibilidades en cuanto al futuro de la Unión existentes en sus Estados miembros. Ciertamente ha sido aprovechada la ocasión por varios de éstos para intentar embridar el proceso de transferencia de competencias soberanas a la Unión, incluso con pretensiones de renacionalización en algún caso, poniéndose límites expresos a cualquier potencial ampliación de las competencias más allá de las atribuidas expresamente, estableciéndose controles más intensos para asegurar la observancia del principio de subsidiariedad y previéndose cláusulas op out en algún caso, que refuerzan la posición de los Estados frente a la tradicional vis expansiva de la Unión. Las excepciones, omisiones, cautelas y contrapesos establecidos con el Tratado de Lisboa, sin embargo y dadas las circunstancias, han sido entendidos por el conjunto de los Estados miembros como el mejor compromiso posible para avanzar conjuntamente y facilitar la salida del impasse que vivía la Unión.

\section{III.2 LA ENTRADA EN VIGOR DEL TRATADO DE LISBOA}

En el apartado III de las consideraciones el Consejo de Estado trata de la entrada en vigor del Tratado de Lisboa regulada en su artículo 6 antes reseñado. La entrada en vigor requiere su ratificación por todos los Estados miembros de la Unión Europea y fija, a modo de término deseable del plazo para depositar los instrumentos y como fecha de entrada en vigor el primer día de 2009. Tal fecha operó como horizonte desde el cual se inicio la cuenta atrás para el cálculo del calendario de elaboración y firma del nuevo Tratado de reforma, puesto que se pretende la entrada en vigor de éste antes de las elecciones al Parlamento Europeo de 2009 y había que tener en cuenta que algunos Estados miembros requieren un período próximo a los doce meses para poder ratificarlo dada la complejidad de sus procedimientos constitucionales internos para la conclusión de un Tratado como éste.

Los Estados que ya han ratificado el Tratado de Lisboa han sido: Hungría (17 de diciembre de 2007), Malta (29 de enero de 2008), Eslovenia (29 de enero de 2008), Rumanía ( 4 de febrero de 2008), Francia ( 7 de febrero de 2008, previa modificación del Título XV de la Constitución de 4 de octubre de 1958 mediante la Ley Constitucional n 2008-103 de 4 de febrero de 2008), Bulgaria (21 de marzo de 2008), Polonia (2 de abril de 2008), Eslovaquia (10 de abril de 2008), Portugal (23 de abril de 2008), Dinamarca y Austria (24 de abril de 2008), Lituania y Letonia (8 de mayo de 2008), Alemania (23 de mayo de 2008), Luxemburgo (29 de mayo de 2008), Grecia, Estonia y Finlandia (11 de junio de 2008) y Gran Bretaña (19 de junio de 2008). En Irlanda, en el referéndum del 12 de junio de 2008, ha triunfado el "no" al Tratado de Lisboa, lo que abre una importante crisis en el proceso de ratificación. 


\section{III.3 El PROCEDIMIENTO DE RATIFICACIÓN DEL TRATADO DE LISBOA}

El apartado IV de las consideraciones se dedica a examinar cuál es el procedimiento de ratificación del Tratado de Lisboa. Comienza expresando que, como se ha dicho en otros dictámenes relativos a la adhesión o ratificación por el Reino de España de los Tratados constitutivos, de reforma y de ampliación de las Comunidades Europeas y de la Unión Europea, el designio de los constituyentes de abrir el ordenamiento español a las influencias del Derecho internacional se manifiesta en varios lugares de la Constitución de 1978 (así, en su preámbulo y en los artículos 10.2 y 96.1). Tal orientación internacionalista alcanza su máxima intensidad en el artículo 93 de la Constitución, antes reseñado. El legislador constitucional tenía la mente puesta en la eventual adhesión de España a las Comunidades Europeas al redactar el artículo 93, pero dicho artículo no se agotó con la adhesión sino que ha conservado su virtualidad para sucesivas aperturas del ordenamiento español al Derecho comunitario: ratificación por España del Acta Unica Europea, del Tratado de la Unión Europea firmado en Maastricht, del Tratado de Amsterdam, del Tratado de Niza, del Tratado de Adhesión a la Unión Europea de diez nuevos Estados miembros, del Tratado de Adhesión a la Unión Europea de la República de Bulgaria y de la República de Rumanía.

El dictamen invoca la Declaración del Tribunal Constitucional 1/2004, de 13 de diciembre de 2004, recaída con carácter previo a la proyectada ratificación por España del Tratado por el que se establece una Constitución para Europa, que formuló diversas consideraciones sobre el alcance y contenido del artículo 93 de la Constitución y que llevan al Consejo de Estado a considerar que el artículo 93 de la Constitución es la "Vía específica cualificada e idónea para que España vaya cubriendo las diversas etapas de la construcción europea, de cuya naturaleza evolutiva era, sin duda, consciente el legislador constitucional». Así se deriva de su naturaleza en cuanto cláusula mediante la que, a través de un mecanismo ad boc previsto por la propia Constitución, se permite verificar una transferencia del ejercicio de competencias derivadas de la Constitución a la Unión sin necesidad de previa reforma constitucional, siempre que tal cesión no traspase los límites materiales inherentes que se derivan de la Constitución. Desde este punto de vista han de someterse a análisis las reformas que introduce el Tratado de Lisboa.

A continuación el Consejo de Estado enumera las innovaciones fundamentales del nuevo Tratado subrayando especialmente el reforzamiento o, cuando menos, la afirmación de la posición de los Estados miembros frente a la Unión a través de numerosas estipulaciones limitativas o previsiones competenciales negativas. El Tratado de Lisboa, así como algunos de los Protocolos y Declaraciones que lo acompañan, han incluido numerosas nuevas cláusulas en defensa de las competencias estatales (por contraste con previsiones del non nato Tratado Constitucional). Destaca las siguientes: 
- La insistencia en el principio de atribución expresa (renumerado artículo 4 del TUE, renumerado artículo 5 del TUE).

- Las disposiciones de la Carta de los Derechos Fundamentales de la UE no ampliarán en modo alguno las competencias de la Unión tal como se definen en los Tratados (artículo 6 del TUE).

- El artículo único del Protocolo 8 en relación con las competencias compartidas.

- La posibilidad de que los proyectos de revisión de los Tratados puedan tener como finalidad reducir las competencias atribuidas a la Unión en los Tratados (artículo 48.2 del TUE). También se prevé expresamente — siguiendo al Tratado Constitucional— la posibilidad de que un Estado miembro se retire de la Unión en cualquier momento, de conformidad con sus normas constitucionales.

- Los procedimientos de revisión no podrán aumentar las competencias atribuidas a la Unión por los Tratados.

- El establecimiento de frenos de emergencia en el ámbito de la cooperación judicial en materia penal (renumerados artículos 82, 83, 86 y 87 del TFUE).

- La Declaración $n^{\circ} 24$, en virtud de la cual la Conferencia confirma que el hecho de que la Unión tenga personalidad jurídica no autorizará en modo alguno a la Unión a legislar o actuar más allá de las competencias que los Estados miembros le han atribuido en los Tratados.

- La potenciación del papel de los Parlamentos Nacionales (artículo 12 renumerado del TUE y Protocolos sobre el cometido de los Parlamentos nacionales en la Unión Europea y sobre la aplicación de los principios de subsidiariedad y proporcionalidad).

A la vista de los aspectos considerados, el Consejo de Estado entiende que el Tratado de Lisboa ofrece un balance equilibrado en el que se perciben notables avances que favorecerán la acción y el funcionamiento de la Unión a través de unas instituciones reforzadas, con mayores competencias, más áreas políticas y procedimientos que permitan funcionar con un elevado número de Estados miembros, al tiempo que las competencias de dichos Estados, en todo aquello que no es objeto de atribución expresa e, incluso, en ámbitos cedidos, son preservadas a través de diversos mecanismos y con distinto grado de intensidad según los casos.

\section{III.4 El aRTículo 93 De la Constitución y el Tratado De LisboA}

Seguidamente el Consejo de Estado analiza el Tratado de Lisboa a la luz del artículo 93 de la Constitución. De su literalidad, tal como expuso el dictamen 2544/2004 (sobre el Tratado Constitucional) se desprenden algunos límites a la posibilidad de que mediante ley orgánica se transfieran competencias derivadas de la Constitución a una organización supranacional como 
es, caracterizadamente, la Unión Europea. Primero, la atribución lo es del ejercicio de competencias y no de su titularidad, advirtiendo, en significativa correlación que el Tratado de Lisboa habilita para que los proyectos de revisión de los Tratados tengan por finalidad reducir las competencias atribuidas a la Unión y considerar el supuesto y efectos de la retirada de los Estados miembros, previsiones que ponen de manifiesto el carácter revocable o recuperable de la cesión.

Segundo, la atribución está referida a las competencias "derivadas de la Constitución", incluido el ejercicio de potestades normativas, ejecutivas y judiciales que comporta "una determinada limitación o constricción, a ciertos efectos, de atribuciones y competencias de los poderes públicos españoles" (Declaración del Tribunal Constitucional de 1 de julio de 1992). El hecho de que con el Tratado de Lisboa la adopción de decisiones por el Consejo en virtud de mayoría cualificada se expanda drásticamente frente a la regla de la unanimidad entraña, sin duda, la aceptación por los poderes públicos españoles de una limitación de sus competencias, pues no tendrán la posibilidad de evitar con su voto actos jurídicos de la Unión que les vincularán en los ámbitos de competencias objeto de transferencia (sin perjuicio de los mecanismos de freno o de exclusión previstos en determinados supuestos, particularmente en las cooperaciones reforzadas).

En tercer lugar, la atribución lo es "de competencias" y no de "las" competencias, de lo que resulta que no cabe una atribución en bloque o global de la totalidad de las competencias que supusiera un vaciamiento de las estatales o la quiebra de la pervivencia de la organización política del Estado (como tampoco una atribución indeterminada de competencias). De un modo especial en este punto el Tratado de Lisboa adoptado por los Estados miembros se ha mostrado muy sensible en aras de contener las suspicacias de algunos de ellos. Así, además de despojarse de los signos estatales y constitucionales que revestían el Tratado Constitucional y que erigían a la Unión como una entidad "en paralelo" a los Estados, el nuevo Tratado insiste a lo largo de su texto en cercenar de manera expresa y a radice eventuales ampliaciones de competencias en manos de las instituciones de la Unión, desarrollando los principios de atribución, de subsidiariedad y de proporcionalidad y obstaculizando así la tradicional expansión indirecta del ámbito común por vías como la doctrina del paralelismo entre las competencias internas y externas de la Comunidad o el principio de efectividad.

En tal sentido, el Consejo de Estado estima que se ha mantenido en el artículo 352 renumerado del TFUE la "cláusula de flexibilidad" establecida en el artículo 308 del TCE y luego en el artículo 1-18 del Tratado Constitucional, en cuya virtud, cuando se considere necesaria una acción de la Unión en el ámbito de las políticas definidas en los Tratados para alcanzar uno de los objetivos fijados por éstos, sin que se hayan previsto en ellos los poderes de actuación necesarios a tal efecto, el Consejo adoptará las disposiciones adecuadas por unanimidad, a propuesta de la Comisión y previa aprobación del Parlamento Europeo. Se trata de un procedimiento perfectamente forma- 
lizado al que, incluso, se han añadido con el Tratado de Lisboa dos nuevas precisiones limitativas: 1. "cuando el Consejo adopte dichas disposiciones con arreglo a su procedimiento legislativo especial, se pronunciará también por unanimidad, a propuesta de la Comisión y previa aprobación del Parlamento Europeo"; 2. "el presente artículo no podrá servir de base para alcanzar objetivos del ámbito de la política exterior y de seguridad común y todo acto adoptado de conformidad con el presente artículo respetará los límites fijados en el párrafo segundo del artículo 40 del Tratado de la Unión Europea". Para más claridad, se insiste en los límites de la Declaración n 42.

Expresa más adelante el Consejo de Estado que, tal como señalara el Tribunal Constitucional en su Declaración 1/2004 respecto del Tratado de la Constitución Europea, cabe afirmar igualmente que el Tratado de Lisboa clarifica y precisa el marco competencial de la Unión y, "en consecuencia, no altera sustancialmente la situación creada tras nuestra adhesión a las Comunidades y, si acaso, la simplifica y reordena en términos que hacen más preciso el alcance de la cesión del ejercicio de competencias verificada por España. Pero, sobre todo, se advierte que las competencias cuyo ejercicio se transfiere a la Unión Europea no podrían, sin quiebra del propio Tratado, servir de fundamento para la producción de normas comunitarias cuyo contenido fuera contrario a valores, principios o derechos fundamentales de nuestra Constitución". Es por otra parte altamente significativo que haya desaparecido del texto del Tratado de Lisboa la regulación del principio de primacía, cuyo tratamiento se encuentra ahora relegado a una Declaración en el Acta Final (la $\mathrm{n}^{\circ}$ 17) que, por lo demás, atempera el tenor del fallido artículo 1-6 del Tratado constitucional, al limitarse a decir matizadamente que la Conferencia recuerda que, con arreglo a la jurisprudencia reiterada del Tribunal de Justicia de la Unión Europea, los Tratados y el Derecho adoptado por la Unión priman sobre el Derecho de los Estados miembros, en las condiciones establecidas por la citada jurisprudencia.

Finaliza el dictamen estimando que, a la luz de las consideraciones expuestas y habida cuenta de los términos expresos de contención en que, según el Tratado de Lisboa, se verificará la transferencia de competencias derivadas de la Constitución por el Reino de España a la Unión Europea, procede concluir que el cauce constitucional idóneo para ratificar dicho Tratado es el previsto en el artículo 93 de la Constitución, puesto que el desplazamiento de las normas constitucionales que atribuyen competencias a los poderes públicos españoles para que pasen a ejercitarlas las instituciones correspondientes a la Unión Europea tiene lugar dentro de la amplia libertad de configuración normativa que deriva de la Constitución española y —en términos de la Declaración del Tribunal Constitucional 1 / 1992 - sin que se advierta que "dispone» de los límites materiales inherentes a ésta, esto, es sin contrariar ni permitir contrariar sus determinaciones.

Por consiguiente, el Consejo de Estado, teniendo en cuenta las innovaciones del Tratado de Lisboa y la Declaración 1/2004, de 13 de diciembre de 2004, del Tribunal Constitucional, no estima necesario acudir a la vía preven- 
tiva prevista en el artículo 95 de la Constitución, a fin de que el Tribunal Constitucional declare si existe o no contradicción entre el Tratado y la Constitución. Resulta de todo ello con claridad que el artículo 93 de la Constitución constituye el cauce debido para la prestación del consentimiento del Estado para obligarse en virtud del Tratado de Lisboa.

De acuerdo con el Consejo de Estado, el 30 de abril de 2008 ha sido presentado en el Congreso de los Diputados un proyecto de Ley Orgánica por la que se autoriza la ratificación por España del Tratado de Lisboa, por el que se modifican el Tratado de la Unión Europea y el Tratado Constitutivo de la Comunidad Europea, firmado en la capital portuguesa el 13 de diciembre de 2007. La Ley Orgánica 1/2008, de 30 de julio, consta de dos artículos, el primero autoriza la ratificación por España del Tratado de Lisboa y el segundo expresa que, a tenor de lo dispuesto en el párrafo segundo del artículo 10 de la Constitución Española y en el apartado 8 del artículo 1 del Tratado de Lisboa, las normas relativas a los derechos fundamentales y a las libertades que la Constitución reconoce se interpretarán también de conformidad con lo dispuesto en la Carta de los Derechos Fundamentales publicada en el Diario Oficial de la Unión Europea de 14 de diciembre de 2007, cuyo texto íntegro reproduce a continuación.

ABSTRACT.- The Council of State analizes firstable, the structure of the new European Treaty and secondly studies its content, it highlights the fact that the Letter of Fundamental Rights of the Union is not incorporate to the text. The Council of State is pronounced on the significance of this new Treaty: in fact the Treaty picks up great part of the Constitutional Treaty, leaving in the road those elements that generated rejection in some States for its excessive constitutional or state connotation. At the end, the Council of State understands that the suitable bed to ratify this new Treaty is the one Foreseen in the article 93 CE. 